\title{
First Experimental Demonstration of Nonlinear Inverse Synthesis Transmission over Transoceanic Distances
}

\author{
S. T. Le*, I. D. Philips, J. E. Prilepsky, P. Harper, N. J. Doran, A. D. Ellis, and S. K. Turitsyn \\ Aston Institute of Photonics Technologies, Aston University, Birmingham, B4 7ET, UK \\ Author e-mail address: let1@aston.ac.uk
}

\begin{abstract}
We demonstrate for the first time, the transmission performance of 10Gbaud nonlinear inverse synthesis based signal over transoceanic distances, showing a significant improvement in data capacity $x$ distance product (x12) compared with other NFT-based systems.
\end{abstract}

\section{Introduction}

Nonlinear Fourier transform (NFT) method and eigenvalue communication [1 - 9] with the use of nonlinear signal spectrum, both discrete [1,3,5-6] and continuous [2, 4, 7 -9], have attracted a great deal of attention recently, as a promising transmission approach to combat impairments caused by fiber nonlinearity. The NFTbased transmission systems explore the fact that the dynamics of individual nonlinear spectral modes are essentially linear. As a result, the nonlinearity-induced cross-talk between them is effectively absent during propagation [1]. Recently, initial experimental demonstrations of NFT-based systems with the use of discrete spectrum have been reported [5,6]. However, the number of transmitted bits per burst and distance reach were limited to 4 bits/burst over $640 \mathrm{~km}$ in [5] and 3 bits/burst over $1800 \mathrm{~km}$ in [6], respectively. One of the key challenges for NFT-based communication is to develop new effective techniques to encode and detect information to/from the signal's nonlinear spectrum. In addition, it is also crucial to develop effective digital signal processing (DSP) techniques to mitigate the impacts other impairments rather than the fiber nonlinearity and possible system's front-end imperfections in NFT-based systems.

In this paper, we report a significant improvement in NFT-based system performance by experimentally demonstrating for the first time, the generation, transmission and detection of a 10Gbaud NFT-based signal (120 bits/burst) over a distance of $7344 \mathrm{~km}$. This represents a factor of 12 improvement in data capacity x distance product compared with other NFT-based systems [5,6]. In this experiment, information is encoded directly onto the continuous part of the signal's nonlinear spectrum using QPSK OFDM waveform via inverse NFT (INFT) and the nonlinear inverse synthesis (NIS) transmission scheme [4, 7 - 9]

\section{Experimental setup}

The schematic of the experimental setup, together with the Tx, Rx DSP are shown in the Fig. 1(a-c), where the green blocks indicate the required additional DSP blocks for NIS transmission in comparison with the conventional schemes. For each burst and each predefined launch power $P_{0}$, a 10Gbaud OFDM waveform (one OFDM symbol, $6 \mathrm{~ns}$ of duration, no cyclic prefix) was generated offline using an IFFT (size of 128), where 60 subcarriers were filled with QPSK data and the remaining subcarriers were set to 0 for oversampling purpose. Guard bands of $12 \mathrm{~ns}$ were added to both the beginning and the end of the OFDM symbol, giving a total burst period of $30 \mathrm{~ns}$. The generated signal was then normalized using the lossless path average (LPA) NLSE model for optical links with lumped amplification [8]. The resulting signal was upsampled (by a factor of 10 times) before being fed into the INFT block. Upsampling is necessary here to reduce the error associated with the INFT. Finally, the output signal after INFT was downsampled to $25 \mathrm{Gs} / \mathrm{s}$ before being loaded into the arbitrary waveform generator (AWG) with 6 bits DAC resolution and fed through a linear amplifier to drive an IQ modulator. As explained in $[4,7,8]$, the INFT maps the linear spectrum of the input signal to the continuous part of the nonlinear spectrum of the output signal. Since the OFDM waveform was used as the input signal of the INFT block, the continuous part of the nonlinear spectrum of the output signal was directly modulated by QPSK data. An example of the transmitted burst is shown in Fig. 1(d).

The transmission experiment used a re-circulating loop consisting of a $4 \times 102 \mathrm{~km}$ span Sterlite OH-LITE (E) fiber ( $19 \mathrm{~dB}$ insertion loss per span) and a leveller. At the receiver, the signal was filtered and amplified (using a low-gain EDFA) before being coherently detected using a real-time $80 \mathrm{Gs} / \mathrm{s}$ sampling oscilloscope. Both the transmitter laser and local oscillator were ECLs each with a linewidth of $\sim 100 \mathrm{kHz}$. The Rx DSP (Fig. 1c) firstly uses a training symbol to perform both timing synchronization and frequency offset compensation. The signal was then separated into a number of discrete $30 \mathrm{~ns}$ bursts before being normalised according to the LPA NLSE model [8].The normalized power $P_{l}$ was adjusted to be slightly different from the actual launch power $P_{0}$ to account for the power variation during each re-circulation resulting from the gain-loss imperfections. After normalization, the forward NFT was performed to recover the continuous part of signal's nonlinear spectrum 


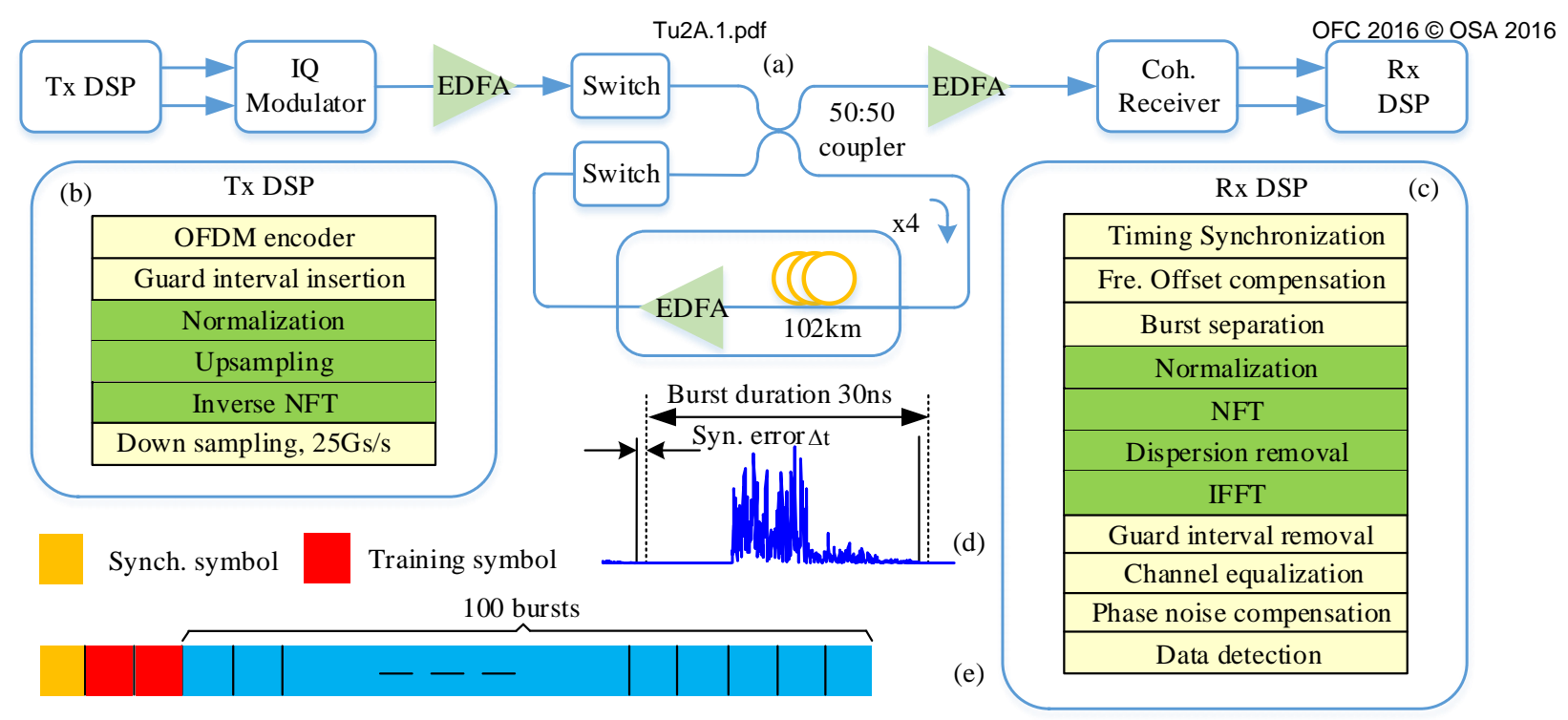

Fig. 1(a): Schematic of the experimental setup of 10 Gbaud NIS-based transmission in fiber link with EDFA-only amplification; (b) - block diagram of the Tx DSP; (c) - block diagram of the Rx DSP; (d) - illustration of a transmitted burst with a duration of 30ns carrying 120 bits (60 QPSK symbols) and illustration of synchronization error, (e) - structure of the transmitted signal, including one synchronization symbol, two training symbols for channel estimation and 100 OFDM NIS-based bursts.

and single-tap dispersion compensation was performed to remove the effects of both the chromatic dispersion and fiber nonlinearity. Next, the IFFT was performed to recover the transmitted time domain signal and then the guard bands were removed. For the NIS-based systems, synchronization error $(\Delta t)$ will result in a frequency dependent phase shift in the nonlinear Fourier domain [3]:

$$
N(q(t-\Delta t), \xi)=e^{-2 j \xi \Delta t} N(q(t), \xi)
$$

where $N(q(t), \xi)$ is the continuous part of the nonlinear spectrum of $q(t)$.

Since the synchronization error is constant for all bursts in one frame, the resulting frequency dependent phase shift can be readily corrected through a single-tap channel estimation and equalization using training sequences. Herein, the first two bursts were used for channel estimation (Fig. 1(e)). The impact of laser phase noise was compensated after channel estimation using 4 pilot subcarriers in each OFDM burst. We corrected for the common phase error only, the impact of which on the NIS-based systems is similar to those of the conventional linear transmission schemes. Finally, the system performance was evaluated directly from the BER by processing 10 recorded traces (each with 100 bursts), and the results are expressed as a $\mathrm{Q}$ factor.

\section{Experimental results and discussion}

The back-to-back performance for different burst power values is presented in Fig. 2 (a). Note that a signal with higher power after INFT requires a higher DAC resolution and longer guard band duration in order to preserve
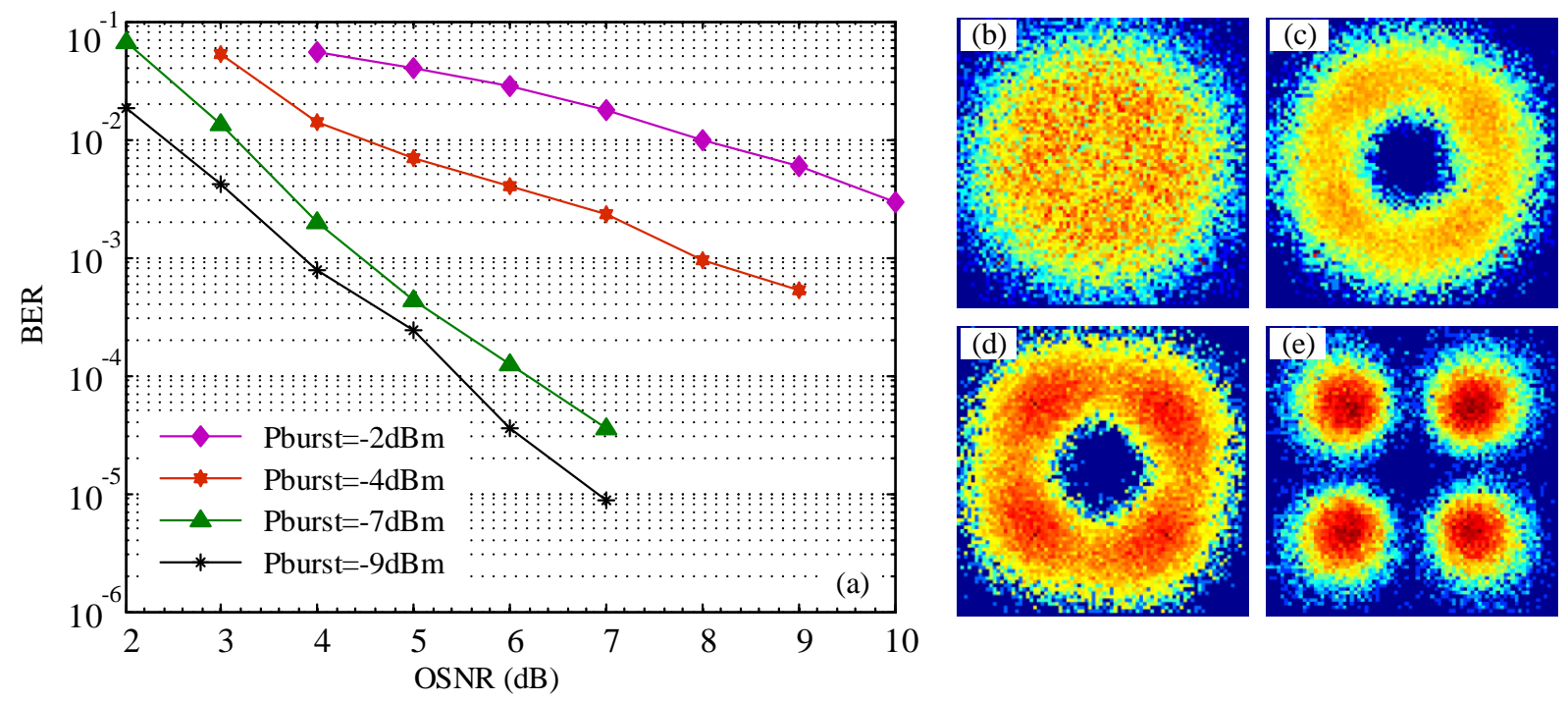

Fig. 2(a): Bit-error-rate performance of 10 Gbaud NIS-based signal for different burst power values. The BER performance of a standard OFDM signal with similar parameters in burst mode is also plotted; Constellation diagrams at the burst power of $-5 \mathrm{dBm}$ after $4080 \mathrm{~km}$ of transmission distance, (b) - before dispersion removal, (c) - before channel estimation, (d) - before phase noise compensation, (e) - final constellation after phase noise compensation 

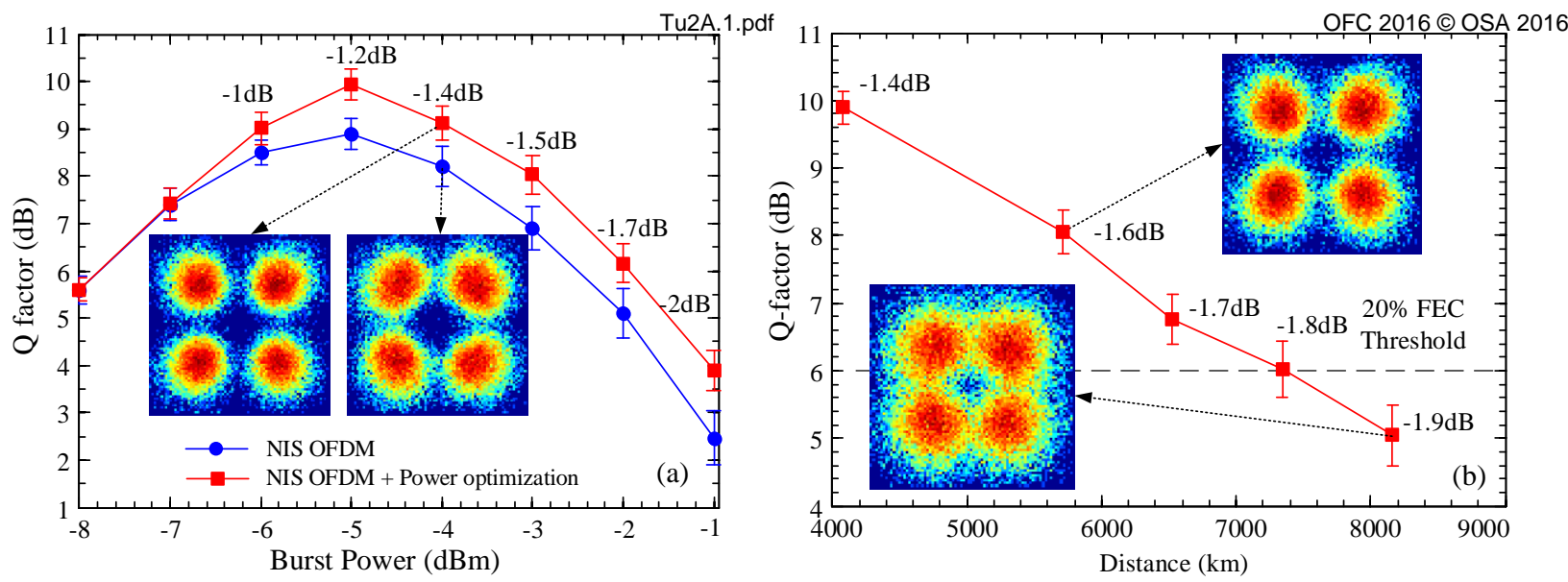

Fig. 3(a). Q-factor as a function of the burst power after $4080 \mathrm{~km}$, the numbers are power correction values for each burst power value; (b) Optimim Q-factor actor as a function of the transmission distance, the numbers are power correction values for each distance value at the optimum burst power.

the longer decaying tail (Fig. 1(d)). As a result, with fixed DAC resolution and guard interval duration, the OSNR penalty increases with the burst power. Typical constellation diagrams after several receiver DSP blocks (single-tap dispersion removal, channel estimation, phase noise estimation) are presented in Fig. 2 (b-e) for the burst power of $-5 \mathrm{dBm}$ after a distance of $4080 \mathrm{~km}$. The final constellation diagram clearly indicates that the transmitted QPSK data was successfully recovered. The system Q-factor is plotted in Fig. 3(a) as a function of the burst power for $4080 \mathrm{~km}$ of distance. The optimum performance was achieved at the burst power of $-5 \mathrm{dBm}$. If the receiver normalized power $P_{1}$ was set to be equal to the launch power $P_{0}$, the optimum Q-factor was found to be $\sim 9 \mathrm{~dB}$ (blue curve). However, by adjusting the normalized power $P_{1}$ an additional $1 \mathrm{~dB}$ gain in $\mathrm{Q}$-factor can be achieved (red curve).

At the launch power of $-5 \mathrm{dBm}$, the optimum value of $P_{1}$ was found to be $-6.2 \mathrm{~dB}\left(P_{0}-1.2 \mathrm{~dB}\right)$. The power correction value in this case was $-1.2 \mathrm{~dB}$. We attribute this phenomenon to the gain-loss imperfection of the loop, which leads to the power variation after each re-circulating loop, degrading the accuracy of the nonlinear pre-distortion technique. The optimum power correction value for each launch power value is also indicated in Fig. 3(a). The system performance degradation in the highly nonlinear regime is due to several contributing factors, including parametric noise amplification and the increasing error associated with the INFT with a fixed DAC resolution and guard interval.

The optimum Q-factor as a function of transmission distance is plotted in Fig. 3(b). The optimum launch power was found to be independent of the transmission distance. After propagation over 18 loops (7344 km) the BER obtained $\left(2.1 \times 10^{-2}\right)$ was below $20 \%$ FEC threshold, indicating the record distance reach of any NFT-based systems up date.

\section{Conclusion}

We have experimentally demonstrated the record distance reach $(7344 \mathrm{~km})$ of any NFT-based systems by encoding and detecting information on/from the continuous part of the nonlinear signal spectrum. Such a demonstration represents a significant advance in the active research area of nonlinear inverse synthesis, and paves the way towards a truly flexible technique that allows the current nonlinear transmission limitations to be exceeded.

Acknowledgement: This work was supported by the UK EPSRC Grant UNLOC (EP/J017582/1). The authors thank Sterlite Technologies and Finisar for their support.

\section{References}

[1] A. Hasegawa and T. Nyu, "Eigenvalue communication,” J. Lightwave Technol. Vol. 11, pp. 395-399 (1993).

[2] E. G. Turitsyna and S. K. Turitsyn, 'Digital signal processing based on inverse scattering transform," Opt. Lett. 38, 4186-4188 (2013)

[3] M. I. Yousefi and F. R. Kschischang, "Information transmission using the nonlinear Fourier transform, Parts I-III," IEEE Trans. Inf. Theory, 60 (2014).

[4] J. E. Prilepsky et al, "Nonlinear Inverse Synthesis and Eigenvalue Division Multiplexing in Optical Fiber Channels", Phys. Rev. Lett. 113, 013901 (2014)

[5] V. Aref et al, "Experimental Demonstration of Nonlinear Frequency Division Multiplexed Transmission," ECOC 15, Valencia, Spain, paper Tu. 1.1.2 (2015).

[6] Z. Dong, et al "Nonlinear Frequency Division Multiplexed Transmissions Based on NFT," IEEE PTL, 27 (2015).

[7] S. T. Le, J. E. Prilepsky, and S. K. Turitsyn, "Nonlinear inverse synthesis for high spectral efficiency transmission in optical fibers," Opt. Express, 22 (2014).

[8] S. T. Le, J. E. Prilepsky, and S. K. Turitsyn, "Nonlinear inverse synthesis technique for optical links with lumped amplification," Opt. Express, 23, 8317-8328 (2015).

[9] S. T. Le et al, "Modified Nonlinear Inverse Synthesis for Optical Links with Distributed Raman Amplification", ECOC 2015, Valencia, Spain, paper Tu 1.1.3 (2015). 